\title{
Holomorphic Versions of the Fabrey-Glimm Representations of the Canonical Commutation Relations*
}

\author{
Judith Kunofsky \\ Department of Mathematics, University of California, Berkeley, California, USA
}

Received September 10, 1974; in revised form December 27, 1974

\section{Introduction}

Glimm and Fabrey have constructed $[6 ; 4]$ a Hilbert space $\mathscr{F}_{r}$ for a simplified version of the $: \Phi^{4}$ : model in quantum field theory for 3 space-time dimensions with space cutoff by using a sequence of truncated exponentials involving $a^{*}(v)$ to define the dressing transformation, where

$$
v\left(k_{1}, k_{2}, k_{3}, k_{4}\right)=\tilde{h}\left(\Sigma k_{i}\right) \Pi \mu\left(k_{i}\right)^{-1 / 2}\left(\Sigma \mu\left(k_{i}\right)\right)^{-1} .
$$

The space cutoff is $h, k_{i} \in \mathbb{R}^{2}, \mu\left(k_{i}\right)=\left(\mu_{0}^{2}+\left|k_{i}\right|^{2}\right)^{1 / 2}$. For $v$ of a more general form, lower parameter $j$, and upper cutoff $\sigma$, they show convergence of $\left(\hat{T}_{j \sigma} \phi, \hat{T}_{j \sigma} \psi\right) e^{-X(\sigma)}$ for $\phi, \psi$ in a dense subset $\mathscr{D}$ of Fock space, as $\sigma \rightarrow \infty$. $\hat{T}_{j \sigma}$ is a truncated version of $e^{a^{*}(v)}$ and $X(\sigma)$ is the renormalization. The closure of the inductive limit of $\hat{T}_{j} \mathscr{D}$ over the lower parameters defines a Hilbert space which carries a Weyl representation of the CCR (canonical commutation relations).

The Bargmann-Segal complex wave representation for the free field has as Hilbert space $H^{2}\left(K_{c x}^{\prime}, d \mu\right)$, the completion of the tame holomorphic functionals on $K_{c x}^{\prime}$, the complex distributions, which are square-integrable with respect to the Gaussian cylinder set measure $\mu$ on $K^{\prime}$. The finite-dimensional case has been discussed by Bargmann [1] and the infinite dimensional case by Segal $[15 ; 16]$. Creation operators on $H^{2}\left(K^{\prime}, d \mu\right)$ are diagonalized and annihilation operators are differentiations.

We construct an analogue to the complex wave representation for the interaction case as a countable inductive limit of spaces of the following form: completion of the tame holomorphic functionals on $K_{c x}^{\prime}$ in the space of functionals which are square integrable with respect to a countably additive measure associated with $T_{j}$. This space carries a representation of the CCR for which creation is a multiplication operator and annihilation is, formally, differentiation plus multiplication by the $\log$ derivative of $T_{j}$. The representation is unitarily equivalent to the Glimm-Fabrey representation.

For a fixed lower parameter $j$ and upper cutoff $\sigma$ we construct $H^{2}\left(K^{\prime}, d \eta_{j \sigma}\right)$, where $d \eta_{j \sigma}=\left|T_{j \sigma}\right|^{2}\left\|T_{j \sigma}\right\|^{-2} d \mu$. In order to show that the $\eta_{j \sigma}$ converge to a countably additive measure, we analyze the characteristic functions $L_{j \sigma}(h)$ of $\eta_{j \sigma}$ and,

\footnotetext{
* This work was supported in part by the National Science Foundation, GP 30798X2.
} 
using estimates derived from Fabrey's analysis and the theory of measures on the dual of a nuclear space, show that $L_{j}(h)=\lim _{\sigma \rightarrow \infty} L_{j \sigma}(h)$ defines a countably additive measure on $K^{\prime}$. We define $H^{2}\left(K^{\prime}, d \eta_{j}\right)$ as the completion of the (tame) polynomials on $K^{\prime}$ in $L^{2}\left(K^{\prime}, d \eta_{j}\right)$. The creation and annihilation operators are "moving weak limits" of corresponding operators on $H^{2}\left(K^{\prime}, d \eta_{j \sigma}\right)$. The creation operator $a_{j}^{*}(h)$ is multiplication by the monomial associated with $h \in K$ and the annihilation operator $a_{j}(h)$ is, formally,

$$
a(h)+\left[\left(a(h) T_{j}\right) / T_{j}\right] \cdot I_{0}
$$

where $a(h)$ is "differentiation in the $h$ direction" and $I_{0}$ is the identity operator.

The Fabrey-Glimm construction takes an inductive limit of the spaces corresponding to different lower parameters in order to get a space on which selfadjointness of the field operator can be demonstrated. We take the inductive limit of the $H^{2}\left(K^{\prime}, d \eta_{j}\right)$ to get a space $\mathscr{H}$ and a representation of the CCR unitarily equivalent to the representation on $\mathscr{F}_{r}$.

Hepp has constructed [7, Chapter 4] a representation on a space obtained by using lower parameter 0 and the Gelfand-Naimark-Segal construction, which is unitarily equivalent to the representation on $\mathscr{F}_{r}$, and therefore also to the representation on $H$.

\section{Background}

\section{A. Finite-dimensional Case (Bargmann Representation)}

The Hilbert space $H^{2}$ is the entire analytic functions of $n$ complex variables, with inner product

$$
(f, g)=\pi^{-n} \int_{C^{n}} f(z) g(z) e^{-\sum\left|z_{i}\right|^{2}} d^{n} z .
$$

Equivalently, this space is the completion of the polynomials on $\mathbb{C}^{n}$ with respect to the Gaussian measure $\pi^{-n} \exp \left(-|z|^{2}\right) d^{n} z$. We define annihilation and creation operators as follows:

$$
\begin{array}{rll}
a_{i}^{*}(f)(z)=z_{i} f(z) & \text { if } & z_{i} f(z) \in H^{2} \\
a_{i}(f)(z)=\partial f / \partial z_{i} & \text { if } & \partial f / \partial z_{i} \in H^{2},
\end{array}
$$

where $\partial f / \partial z_{i}=1 / 2\left(\partial f / \partial x_{i}-i \partial f / \partial y_{i}\right)$. The operators $a_{i}^{*}(f)$ and $a_{i}(f)$ are closed, adjoints, and $\left[a_{i}, a_{j}^{*}\right] f=\delta_{i j} f$.

\section{B. Infinite Dimensional Case (Fock Representation)}

As usual, Fock space is $\mathscr{F}=\bigoplus_{n=0}^{\infty} \mathscr{F}_{n}$, where

$\mathscr{F}_{0}=\mathbb{C}$.

$\mathscr{F}_{n}=S L_{2}\left(\mathbb{R}^{2 n}\right)$, the symmetric, square integrable functions of $2 n$ variables, written as functions of $k_{1}, \ldots, k_{n}, k_{i} \in \mathbb{R}^{2}$.

$$
\phi=\Sigma \phi_{n} \in \mathscr{F} \quad \text { if } \quad \sum_{n=0}^{\infty}\left\|\phi_{n}\right\|^{2}=\|\phi\|^{2}<\infty .
$$


$\mathscr{D}$ will denote the subset of $\mathscr{F}$ consisting of $\phi=\Sigma \phi_{n}$ such that only finitely many $\phi_{n}$ are non zero and each $\phi_{n}$ has compact support.

For $f \in L_{2}\left(\mathbb{R}^{2}\right)$,

$$
\begin{aligned}
a^{*}(f) \phi_{n} & =(n+1)^{1 / 2} S\left(f \otimes \phi_{n}\right) \\
\left(a(f) \phi_{n}\right)\left(k_{1}, \ldots, k_{n-1}\right) & =n^{1 / 2} \int f\left(k_{n}\right) \phi_{n}\left(k_{1}, \ldots, k_{n}\right) d k_{n}
\end{aligned}
$$

where $\phi_{n} \in \mathscr{F}_{n}$ and $S$ is the symmetrization operator

$$
\left(S \phi_{n}\right)\left(k_{1}, \ldots, k_{n}\right)=\frac{1}{n !} \sum_{\sigma} \phi_{n}\left(k_{\sigma(1)}, \ldots, k_{\sigma(n)}\right) .
$$

$a^{*}(f)$ and $a(\bar{f})$ are adjoints and $\left[a(f), a^{*}(g)\right]=(f, g)$.

$K(a)$ will be the space of infinitely-differentiable complex-valued functions on $\mathbb{R}^{2}$, with support in $\{\|x\| \leqq a\}$. A sequence $\left\{f_{n}\right\}$ converges to 0 in $K(a)$ if the $f_{n}$ and all their derivatives converge uniformly to 0 . $K$, the inductive limit of the $K(a)$ is usually denoted elsewhere by $\mathscr{D}\left(\mathbb{R}^{2}\right)$.

An element of the form $a^{*}\left(f_{1}\right) \ldots a^{*}\left(f_{s}\right) \Omega_{0}$, where the $f_{i}$ 's $\in K$ and need not be distinct, is called a monomial on $\mathscr{F}$. If $\phi=\Sigma \phi_{i}$, where each $\phi_{i}$ is a monomial and the sum is finite, then $\phi$ is called a polynomial on $\mathscr{F}$ and will be denoted by $\hat{p} \Omega_{0}$. In particular, $\hat{p} \Omega_{0} \in \mathscr{D}$. $\hat{p}$ will be called a polynomial operator.

\section{Infinite-dimensional Case (Bargmann-Segal Representation)}

The coordinate functions $z_{i}$ of the Bargmann representation will correspond to elements of $K^{\prime}$ and analytic functions of $z$ will correspond to the completion of polynomials on $K^{\prime}$ with respect to $L^{2}$ of the infinite dimensional analogue of Gaussian measure.

A function $\psi$ defined on $K^{\prime}$ is based on $F$, where $F$ is a finite dimensional, closed (complex) subspace of $K$, if there is a function $\psi_{1}$ defined on $K^{\prime} / F^{\circ}$ such that $\psi(q)=\psi_{1}(\pi q)$, where $\pi$ is the natural projection: $K^{\prime} \rightarrow K^{\prime} / F^{\circ}$. A function $\psi$ on $K^{\prime}$ is a monomial if there is an $F$, as above, $f_{1}, \ldots, f_{n} \in F, m_{1}, \ldots, m_{n} \in Z^{+}$and a complex constant $\alpha$, such that $\psi$ can be written as

$$
\psi(q)=\alpha\left\langle f_{1}, \pi q\right\rangle^{m_{1}} \ldots\left\langle f_{n}, \pi q\right\rangle^{m_{n}} .
$$

A polynomial is a finite sum of monomials.

Notation and Comments. (a) $\langle f, q\rangle$ means $f \in K, q \in K^{\prime}$ and the bracket denotes evaluation.

(b) Since $F^{\prime}$ is isomorphic to $K^{\prime} / F^{\circ}$, the second element in the bracket can equivalently be an element of $K^{\prime} / F^{\circ}$, when $f \in F$.

(c) If $i$ denotes the natural injection of $K$ into $K^{\prime}$, we have $\langle f, i(h)\rangle=(h, f)$, where parentheses denote the inner product on $K$, and the inner product is complex linear in the second variable.

(d) $\mu$ denotes the Gaussian measure on $K^{\prime}$ induced by the inner product on $K$.

(e) $H^{2}\left(K^{\prime}, d \mu\right)=_{\text {df. }}$ completion of the polynomials in $L^{2}\left(K^{\prime}, \mu\right)$.

(f) A polynomial on $K^{\prime}$ will be denoted by $p$, with or without subscripts and superscripts. 
Example. Suppose

$$
\psi(q)=\alpha\left\langle f_{1}, \pi q\right\rangle^{2}\left\langle f_{2}, \pi q\right\rangle^{3} .
$$

and $f_{1}$ and $f_{2}$ are orthonormal. Then

$$
\int_{K^{\prime}} \psi(q) d \mu(q)=\pi^{-2} \int_{\mathbb{C}^{2}} \alpha \bar{\lambda}_{1}^{2} \bar{\lambda}_{2}^{3} \exp \left(-\left|\lambda_{1}\right|^{2}-\left|\lambda_{2}\right|^{2}\right) d \lambda_{1} d \lambda_{2} .
$$

A polynomial on $K^{\prime}$ corresponds to an anti-polynomial on $F$, so the representation induced on $F$ will be an anti-holomorphic representation of the CCR.

$H^{2}\left(K^{\prime}, d \mu\right)$ is isomorphic to Fock space, if we define the map $I: \mathscr{F} \rightarrow H^{2}\left(K^{\prime}, d \mu\right)$ as follows: $I \Omega_{0}=1$, the identity function. If $f_{1}, \ldots, f_{s} \in K, I\left(a^{*}\left(f_{1}\right) \ldots a^{*}\left(f_{s}\right) \Omega_{0}\right)$ $=\left\langle f_{1}, \cdot\right\rangle \ldots\left\langle f_{s}, \cdot\right\rangle$. Finite sums of expressions of this form are dense in $\mathscr{F}$ so since the map thus far defined is an isometry, it can be extended to the closures. In particular, $I\left(\hat{p} \Omega_{0}\right)=p$.

Annihilation and creation operators on $H^{2}\left(K^{\prime}, d \mu\right)$, for $f \in K$, can be defined via the isomorphism. In particular, if $\psi \in H^{2}\left(K^{\prime}, d \mu\right)$,

$$
a_{H}^{*}(f) \psi=\langle f, \cdot\rangle \psi
$$

if the right-hand side is in $H^{2}$ and

$$
a_{H}(f)\langle g, \cdot\rangle^{m}=m(\bar{f}, g)\langle g, \cdot\rangle^{m-1} .
$$

$a_{H}^{*}$ is a multiplication operator and $a_{H}(f)$ is "differentiation in the $f$ direction".

Both are closed, $\left[a_{H}(f)\right]^{*} \supset a_{H}^{*}(\bar{f})$, and $\left[a_{H}(f), a_{H}^{*}(g)\right]=(\bar{f}, g) I_{0}, I_{0}=$ identity operator on $H^{2}\left(K^{\prime}, d \mu\right)$, because the corresponding statements are true in Fock space.

Segal's description of the holomorphic representation of the CCR may be found in [15] and [16].

From now on, $a$ and $a^{*}$ will denote operators on $\mathscr{F}$ or $H^{2}\left(K^{\prime}, d \mu\right)$, depending on the context.

\section{The Interaction Case (Renormalized Fock Space $\mathscr{F}_{r}$ )}

$\mathscr{F}_{r}$ is defined using expressions of the form $\lim _{\sigma \rightarrow \infty}\left(\hat{T}_{k \sigma} \psi, \hat{T}_{k \sigma} \psi\right) e^{-X(\sigma)}$ where $\hat{T}_{k \sigma}$ is a truncated version of $e^{a^{* 4}(v)}$ and $X(\sigma)=4 !\left(v_{\sigma}, v_{\sigma}\right), v_{\sigma}$ satisfying certain growth conditions:

We deal with symmetric, measurable functions $v\left(k_{1}, k_{2}, k_{3}, k_{4}\right)$ which are "almost in $L_{2}$ ", i.e. satisfy certain growth conditions. The class of "almost in $L_{2}$ " functions does not contain $L_{2}$, but does contain

$$
\left\{v \in L_{2}\left(\mathbb{R}^{8}\right): \prod_{i=1}^{4}\left(\mu_{0}^{2}+\left|k_{i}\right|^{2}\right)^{\varepsilon / 2} v \in L_{2}\left(\mathbb{R}^{8}\right)\right\} .
$$

The particular $v$ 's arising in field theory are "almost in $L_{2}$ " but not in $L_{2}$. 
Choose $\alpha>1, n(k)$ strictly increasing but polynomial bounded, and construct $\hat{T}_{j \sigma}$ as follows [we write $\hat{T}_{j \sigma}$ for Fabrey's $T_{j \sigma}$, etc.; $\left.k=\left(k_{1}, k_{2}, k_{3}, k_{4}\right)\right]$ :

$$
v_{\varrho \sigma}(k)=\left\{\begin{array}{cc}
v(k) & \max _{1 \leqq i \leqq 4}\left|k_{i}\right| \in[\varrho, \sigma) \\
0 & \text { otherwise } .
\end{array}\right.
$$

Let $v_{\sigma}=v_{0 \sigma} ;$ let $\alpha(j)=\left\{\begin{array}{lll}\alpha^{j} & \text { if } j \geqq 1 \\ 0 & \text { if } j=0\end{array}\right.$.

Let $v_{j \sigma}$ and $v_{j k}$ denote $v_{\alpha(j) \sigma}, v_{\alpha(j) \alpha(k)}$, respectively. Suppose $v_{\sigma} \in L_{2}\left(\mathbb{R}^{8}\right), j \leqq l$, and $\alpha(l) \leqq \sigma \leqq \alpha(l+1)$.

We are examining situations involving "fourth-order" creation operators. Suppose $w=w\left(k_{1}, \ldots, k_{4}\right) \in L_{2}\left(\mathbb{R}^{8}\right)$. Define $a^{* 4}(w)$ as a map from $\mathscr{F}_{n}$ to $\mathscr{F}_{n+4}$ by

$$
a^{* 4}(w) \psi_{n}=[(n+1) \ldots(n+4)]^{1 / 2} S\left(w \otimes \psi_{n}\right)
$$

where $S$ is the symmetrization operator and $\psi_{n} \in \mathscr{F}_{n}$. Operators of this kind form the most singular part of the field operator $: \phi^{4}$ :

In particular,

$$
\begin{gathered}
V_{j \sigma}=\left\{\begin{array}{cc}
a^{* 4}\left(v_{j, j+1}\right) & j \leqq l-1 \\
a^{* 4}\left(v_{l \sigma}\right) & j=l
\end{array}\right. \\
\exp _{n}(x)=\sum_{l=0}^{n} x^{l} / l !
\end{gathered}
$$

Then $\hat{T}_{j \sigma}=\prod_{j_{0} \leqq j \leqq l} \exp _{n(j)} V_{j \sigma}$, and is defined on $\mathscr{D}$.

Theorem (Fabrey). For $\phi, \psi \in \mathscr{D}$ the limit $\lim _{\sigma \rightarrow \infty}\left(\hat{T}_{k \sigma} \phi, \hat{T}_{l \sigma} \psi\right) e^{-X(\sigma)}=\left(\hat{T}_{k} \phi, \hat{T}_{l} \psi\right)_{r}$ exists. If $k \geqq l, \sigma \geqq \alpha(k)$, then $\hat{T}_{l \sigma} \psi=\hat{T}_{k \sigma} \theta$, where

$$
\theta=\prod_{j=l}^{k-1} \exp _{n(j)} \hat{V}_{j \sigma} \psi \in \mathscr{D} .
$$

$(\cdot, \cdot)_{r}$ provides a positive definite inner product for $\bigcup_{j \geqq 0} \hat{T}_{j} \mathscr{D}$, whose completion is denoted $\mathscr{F}_{r}$. Operators $W_{r}(f)=e^{i \phi_{r}(f)}$ can be defined on $\mathscr{F}_{r}$ and satisfy the Weyl form of the CCR.

E. The Interaction Case (Renormalized Bargmann-Segal Space)

We would like to show that the Fabrey-Glimm expressions

$$
\left(\hat{T}_{k} \hat{p}_{1} \Omega_{0}, \hat{T}_{k} \hat{p}_{2} \Omega_{0}\right)_{r}=\lim _{\sigma \rightarrow \infty}\left(\hat{T}_{k \sigma} \hat{p}_{1} \Omega_{0}, \hat{T}_{k \sigma} \hat{p}_{2} \Omega_{0}\right) e^{-X(\sigma)}
$$

can be written as

$$
\int_{K^{\prime}} c_{k}^{-2} \bar{p}_{1} p_{2} d \eta_{k}(\cdot)
$$

where $\eta_{k}$ is a countably additive normalized measure on $K^{\prime}$. To do this we use criteria for a function on $K$ to be the characteristic function of a countably additive measure on $K^{\prime}$. 


\section{Characteristic Functions and Measures on K'}

Theorem III.1 (see [13] and [11]). Let E be a complex locally convex topological vector space. Suppose $L$ is a complex-valued function defined on $E$ with the properties that:

(a) $L(0)=1$.

(b) $L$ is positive definite, i.e., let $g_{1}, \ldots, g_{s} \in E, \xi_{1}, \ldots, \xi_{s} \in \mathbb{C}$. Then

$$
\sum_{i, j=1}^{s} L\left(g_{i}-g_{j}\right) \bar{\xi}_{i} \xi_{j} \geqq 0
$$

(c) L continuous on finite dimensional subspaces of $E$, i.e. suppose $\left\{g_{n}\right\} \subset F$ $=$ space generated by $f_{1}, \ldots, f_{s} \in E, g_{n}=\sum_{i=1}^{s} a_{i}^{(n)} f_{i}$. Then $\lim _{n \rightarrow \infty} a_{i}^{(n)}=0, i=1, \ldots, s$ $\Rightarrow L\left(g_{n}\right) \rightarrow L(0)=1$.

Then $L$ is the characteristic function of some cylinder-set measure $\eta$ (possibly not countably additive) and

And the converse is true.

$$
L(g)=\int_{E^{\prime}} e^{i \operatorname{Re}\langle g, \cdot\rangle} d \eta(\cdot)
$$

$\eta$ is said to satisfy the continuity condition if, for each bounded continuous function $B$ on $\mathbb{C}^{n}$, the function

$$
\Phi\left(f_{1}, \ldots, f_{n}\right)=\int_{E^{\prime}} B\left(\left\langle f_{1}, \cdot\right\rangle, \ldots,\left\langle f_{n}, \cdot\right\rangle\right) d \eta(\cdot)
$$

is sequentially continuous.

If $E$ is a countably Hilbert (complex) nuclear space, for example $K(a)$, then any positive, normalized cylinder set measure $\eta$ in $E^{\prime}$, satisfying the continuity condition, is countably additive ([5], extended to the complex case).

For our purposes the following equivalent version of the continuity condition will be more useful:

For any $A>0$, and any sequence $\left\{g_{j}\right\}$ converging to 0 in $E$ we have $\lim _{j \rightarrow \infty} \eta\left\{q:\left|\left\langle g_{j}, q\right\rangle\right| \geqq A\right\}=0[12]$.

If we are given a function $L$ on all of $K$ satisfying the hypotheses of the above theorem, and whose associated $\eta$ satisfies the continuity condition, we can construct a countably additive measure on $K^{\prime}=(\cup K(a))^{\prime}$ and extend the measure to the Borel sets of $K^{\prime}$.

Example 1. Suppose $p$ is a polynomial on $K^{\prime}$. Then $p \in L^{2}\left(K^{\prime}, d \mu\right)$ since polynomials are square integrable with respect to Gaussian measure. Define

$$
\begin{aligned}
L(g) & =\left(p e^{-i / 2\langle g, \cdot\rangle}, p e^{i / 2\langle g, \cdot\rangle}\right)\|p\|^{-2} \text { with inner product in } K^{\prime}, \\
& =\int_{K^{\prime}} \bar{p} e^{i / 2 \overline{\langle g, \cdot\rangle}} p e^{i / 2\langle g, \cdot\rangle}\|p\|^{-2} d \mu(\cdot)=\int_{K^{\prime}} e^{i \operatorname{Re}\langle g, \cdot\rangle}|p|^{2}\|p\|^{-2} d \mu .
\end{aligned}
$$

Then $L$ satisfies the hypotheses of Theorem III.1 and the continuity condition, and hence defines a countably additive measure $\eta_{p}$. 
Example 2. More generally, suppose $T \in L^{2}\left(K^{\prime}, d \mu\right),\|T\| \neq 0$. Then

$$
\eta(Z)=\int_{Z} \frac{|T|^{2} d \mu}{\|T\|^{2}}
$$

defines a countably additive measure on the $\sigma$-algebra generated by the cylinder sets.

Our goal is to show initially that $I\left(\hat{T}_{k \sigma} \Omega_{0}\right) \in L^{2}\left(K^{\prime}, d \mu\right)$, and then that the corresponding $\eta_{k \sigma}$ converge to a measure $\eta_{k}$ which is associated with the $\left(\hat{T}_{k} \phi, \hat{T}_{k} \psi\right)_{r}$.

Suppose $T \in H^{2}\left(K^{\prime}, d \mu\right)$ has the property that $I^{-1} T$ is of the form $\hat{T} \Omega_{0}$, where $\hat{T} \Omega_{0}$ is a finite sum of terms of the form $a^{* 4}\left(f_{1}\right) \ldots a^{* 4}\left(f_{j}\right), f_{i} \in L^{2}\left(\mathbb{R}^{8}\right)$.

$\hat{T}$ is a bounded operator on $\bigoplus_{n \leqq N} \mathscr{F}_{n}$ for any $N$, and this property forms the key to proofs of the lemmas below.

Lemma III.2. $I(\hat{T})$, the corresponding operator on $H^{2}\left(K^{\prime}, d \mu\right)$, is multiplication by the function T, i.e. $M_{T}$. For example, $I(\hat{p})=M_{p}$.

Lemma III.3. $T \cdot p \in H^{2}\left(K^{\prime}, d \mu\right)$ for all polynomials $p$. In particular, $I\left(\hat{T} \Omega_{0}\right)=T$.

Lemma III.4. We can define isometric isomorphisms $E$ and $E_{T}$ with

$$
\left\{\hat{T} \hat{p} \Omega_{0}\right\}^{-} \underset{I}{\longrightarrow}\left(H^{2}\left(K^{\prime}, d \mu\right) \cap\{T \cdot p\}\right)^{-} \underset{E}{\longrightarrow} H^{2}\left(K^{\prime}, d \eta_{T}\right)
$$

by setting

$$
\begin{aligned}
E(T \cdot p) & =\left\|\hat{T} \Omega_{0}\right\| \cdot p \\
E_{T} & =E \circ I
\end{aligned}
$$

and extending the domains to their closures in $L^{2}\left(K^{\prime}, d \mu\right)$ and $\mathscr{F}$ respectively. In particular,

$$
\left(\hat{T} \hat{p}_{1} \Omega_{0}, \hat{T} \hat{p}_{2} \Omega_{0}\right)\left\|\hat{T} \Omega_{0}\right\|^{-2}=\int_{K^{\prime}} \bar{p}_{1} p_{2} d \eta_{T} .
$$

Lemma III.5. Suppose $\psi=\Sigma \psi_{n} \in \bigoplus_{n \leqq N} \mathscr{F}_{n}$ for some $N$. Then $\hat{T} \psi \in\left\{\hat{T} \hat{p} \Omega_{0}\right\}^{-}$and so corresponds to an element $E_{T}(\hat{T} \psi)$ of $H^{2}\left(K^{\prime}, d \eta_{T}\right)$.

We define $a_{T}^{*}(f), a_{T}(f)$ on $H^{2}\left(K^{\prime}, d \eta_{T}\right)$ using the isomorphism $E$.

Let $\phi \in H^{2}\left(K^{\prime}, d \eta_{T}\right)$.

Then

and

$$
a_{T}^{*}(f) p=E a^{*}(f) T \cdot p /\|T\|=E(T\langle f, \cdot\rangle p /\|T\|)=\langle f, \cdot\rangle p
$$

$$
a_{T}(f) p=E a(f) T \cdot p /\|T\|=E[T \cdot(a(f) p)+(a(f) T) \cdot p] /\|T\|
$$

which might not be in the domain of $E$ so we do not know if $a_{T}(f)$ can be defined on the polynomials.

Formally, we would get

$$
=a(f) p+p \cdot " a(f) T " / T=\left(a(f)+(" a(f) T " / T) I_{0}\right) p
$$

where $I_{0}$ is the identity operator.

If $a(f) T=0$, then $a_{T}(f) p$ is well defined, as the usual derivative. 
We can consider the bilinear form $A_{T}$ defined by

$$
\left(p_{1}, A_{T} p_{2}\right)_{T}=_{\text {dt. }}\left(E^{-1} p_{1}, A E^{-1} p_{2}\right)
$$

for a bilinear form $A$.

If we let $A_{T}$ correspond to $A=\left[a(f), a^{*}(g)\right]$, then formally,

$$
\begin{aligned}
\left(p_{1},\left[a_{T}(f), a_{T}^{*}(g)\right]\right)_{T} “ & ="\left(p_{1}, A_{T} p_{2}\right)_{T} \\
& =\left(E^{-1} p_{1},\left[a(f), a^{*}(g)\right] E^{-1} p_{2}\right) \\
& =\left(E^{-1} p_{1},(\bar{f}, g) E^{-1} p_{2}\right) \\
& =(\bar{f}, g)\left(p_{1}, p_{2}\right)_{I}
\end{aligned}
$$

where we have assumed that the domain of $\left[a(f), a^{*}(g)\right]$ includes $E^{-1} p_{1} \times E^{-1} p_{2}$ and used the commutation relations on $H^{2}\left(K^{\prime}, d \mu\right)$. This can be called a weak representation of the CCR.

The Fabrey-Glimm construction considers $T$ 's which are truncated exponentials, i.e., $T \sim e^{\langle f, \cdot\rangle^{4}}$. So formally,

$$
(a(g) T) / T=e^{-\langle f, \cdot\rangle^{4}} \cdot 4\langle f, \cdot\rangle^{3} e^{\langle f, \cdot\rangle^{4}}(\bar{g}, f)=4(\bar{f}, g)\langle f, \cdot\rangle^{3},
$$

an expression which makes sense.

Theorem III.6. $I\left(\hat{T}_{k \sigma} \Omega_{0}\right)=T_{k \sigma} \in H^{2}\left(K^{\prime}, d \mu\right)$ satisfies the hypotheses of Lemma III.2 and so is an instance of Example 2 and the succeeding discussion.

In particular,

$$
\left(\hat{T}_{k \sigma} \hat{p}_{1} \Omega_{0}, \hat{T}_{k \sigma} \hat{p}_{2} \Omega_{0}\right)\left\|\hat{T}_{k \sigma} \Omega_{0}\right\|^{-2}=\int_{\bar{K}^{\prime}} \bar{p}_{1} p_{2} d \eta_{k \sigma}
$$

where $\eta_{k \sigma}$ is a countably additive cylinder set measure of total mass 1 .

Also

$$
L_{k \sigma}(f)={ }_{\mathrm{d} t .} \int_{K^{\prime}} e^{i \operatorname{Re}\langle f \cdot \cdot\rangle} d \eta_{k \sigma}(\cdot)
$$

is positive definite.

This expression differs from the expressions whose limits define $\mathscr{F}$, by a factor $\left(\hat{T}_{k \sigma} \Omega_{0}, \hat{T}_{k \sigma} \Omega_{0}\right) e^{-X(\sigma)}$. We show below that as $\sigma \rightarrow \infty$ this factor converges to a constant $c_{k}^{-2}$, where $0<c_{k}^{-2}<1$.

\section{Some Fundamental Estimates}

In order to show that the Fabrey-Glimm expressions of the form $\left(\hat{T}_{k} \hat{p}_{1} \Omega_{0}\right.$, $\left.\hat{T}_{k} \hat{p}_{2} \Omega_{0}\right)_{r}$ can be written as $\int_{\boldsymbol{K}^{\prime}} \bar{p}_{1} p_{2} d \eta_{k}(\cdot)$, where $\eta_{k}$ is a countably additive measure on $K^{\prime}$, we examine

$$
L_{k}(f)={ }_{\text {df. }} \lim _{\sigma \rightarrow \infty} L_{k \sigma}(f)={ }_{\text {dt. }} \lim _{\sigma \rightarrow \infty}\left(\hat{T}_{k \sigma} e^{-i / 2 a^{*}(f)} \Omega_{0}, \hat{T}_{k \sigma} e^{i / 2 a^{*}(f)} \Omega_{0}\right)\left\|\hat{T}_{k \sigma} \Omega_{0}\right\|^{-2}
$$


where $e^{a^{*}(g)}$ is defined as $\sum_{k=0}^{\infty} a^{*}(g)^{k} / k$ !, and show it is a characteristic function. Even $\hat{T}_{k \sigma} e^{-i / 2 a^{*}(f)} \Omega_{0}$ has an infinite number of particles, so the existence of $L_{k \sigma}$ needs to be shown.

Using the isomorphism between $\mathscr{F}$ and $H^{2}\left(K^{\prime}, d \mu\right)$ we can write

$$
L_{k \sigma}(f)=\int_{K^{\prime}} e^{i \operatorname{Re}\langle f, .\rangle} d \eta_{k \sigma} .
$$

The operator $e^{a^{*}(g)}$, for $g \in K$, defines a bounded map from $\mathscr{F}_{n}$ to $\mathscr{F}$, for any $n$.

Now $\left(\hat{T}_{k \sigma} \phi, \hat{T}_{k \sigma} \psi\right) e^{-X(\sigma)}=\left(\phi, \hat{T}_{k \sigma}^{*} T_{k \sigma} \psi\right) e^{-X(\sigma)} . \hat{T}_{k \sigma}^{*} \hat{T}_{k \sigma}$ is a truncated power series in $V_{\sigma}^{*}$ and $V_{\sigma}$ and can be rewritten as a sum of Wick-ordered terms with distribution kernels by using the commutation relations. Fabrey calls a term reduced if it contains no $X=V^{*}{ }_{4}^{-}-V$ components, i.e. no completely contracted terms.

Fabrey shows that $\left(\phi, \hat{T}_{k \sigma}^{*} \hat{T}_{k \sigma} \psi\right) e^{-X(\sigma)}=\int_{E} h_{\sigma}$, where $h_{\sigma}$ is a measurable function on the space $E$, and $E$ is a direct sum of spaces associated with reduced terms in $\hat{T}_{k \sigma}^{*} \hat{T}_{k \sigma}$.

The following lemma is Fabrey's Lemma 3.3, with several components of the constant exhibited explicitly.

Lemma IV.1. Suppose $G$ is a reduced graph such that $|G|=n, \phi$ and $\psi \in \mathscr{D}_{i}, \mathscr{D}_{j}$, respectively, and $\phi, \psi$ vanish off a sphere of radius $\varrho$. Then

$$
\left(|\phi|,\left|R_{0}\right||\psi|\right) \leqq K_{1} K_{2}^{n} \alpha^{-\varepsilon c n^{1+\delta}}\|\phi\|\|\psi\|\left(\mu_{0}^{2}+\varrho^{2}\right)^{a(i+j) / 2}(j+1)^{2 n}
$$

for constants $K_{1}, K_{2}$.

Lemma IV.2. (existence of $\left.\left.\hat{T}_{k} e^{a^{*}(f)} \Omega_{0}, \hat{T}_{k} e^{a^{*}(g)} \Omega_{0}\right)_{r}\right)$. Suppose $f, g \in L^{2}\left(\mathbb{R}^{2}\right)$ and $f(k)=g(k)=0$ for $\|k\|>\varrho>0$. Then

$$
\lim _{\sigma \rightarrow \infty}\left(\hat{T}_{k \sigma} e^{a^{*}(f)} \Omega_{0}, \hat{T}_{k \sigma} e^{a^{*}(g)} \Omega_{0}\right) e^{-X(\sigma)}<\infty
$$

and there is a uniform bound for the expressions corresponding to those f's and g's with $\|f\|<\delta,\|g\|<\delta$ for some $\delta>0$.

Proof. The proof consists of several parts:

A) expressing the inner product as $\int_{E} h_{\sigma}$ where $E$ is a measure space; showing the $h_{\sigma}$ converge pointwise and $\left|h_{\sigma}\right| \leqq h$ where $h$ is measurable on $E$ ([3], Lemmas $3.1,3.2$, and corollary),

B) estimating $\left(|\phi|,\left|R_{0}\right||\psi|\right)$ for a reduced term $R_{0}$,

C) writing $\int h$ as a sum, $\sum_{i, j, n=0}^{\infty}$, of expressions of the form described in B),

D) summing over $i$,

E) over $j$,

F) over $n$,

G) applying the dominated convergence theorem to get convergence of $\int_{E} h_{\sigma}$ as $\sigma \rightarrow \infty$. 
B) Let $\phi_{i}=a^{*}(f)^{i} \Omega_{0} / i$ ! Then $\left\|\phi_{i}\right\| \leqq(i !)^{-1 / 2}\|f\|^{i}$.

Let $R_{0}$ be a particular reduced term with graph $G$ and $|G|=n$. Then $\left(|\phi|,\left|R_{0}\right||\psi|\right)$

$\leqq \sum_{i, j=0} K_{1} K_{2}^{n} \alpha^{-\varepsilon c n^{1+\delta}}\left(\mu_{0}^{2}+\varrho^{2}\right)^{a j / 2}(j+1)^{2 n}\|g\|^{j}(j !)^{-1 / 2}\left(\mu_{0}^{2}+\varrho^{2}\right)^{a i / 2}\|f\|^{i}(i !)^{-1 / 2}$.

C) We want to look at

$$
\int_{E} h=\sum_{n} \sum_{\substack{G \ni \\|G|=n}} \sum_{i, j}\left(\left|\phi_{i}\right|,\left|R_{0}\right|\left|\psi_{j}\right|\right) .
$$

Lemma IV.1 estimates the inner products and Fabrey's Lemma 3.4 says there are at most $K_{3}^{n}(4 n) !^{2}$ reduced graphs $G$ such that $|G|=n$, where $K_{3}$ is constant. So we need to examine

$$
\begin{gathered}
K_{1} \sum_{n} K_{2}^{n} K_{3}^{n}(4 n) !^{2} \alpha^{-\varepsilon c n^{1+\delta}} \sum_{j=0}^{\infty}\left(\mu_{0}^{2}+\varrho^{2}\right)^{a j / 2}(j+1)^{2 n}\|g\|^{j}(j !)^{-1 / 2} \\
\cdot \sum_{i=0}^{\infty}\left(\mu_{0}^{2}+\varrho^{2}\right)^{a i / 2}\|f\|^{i}(i !)^{-1 / 2}
\end{gathered}
$$

D) The sum over $i$ converges by the ratio test. In fact if $\|f\|<\delta$, there will be a uniform bound on the sum.

E) Fix $n$. The sum we want to estimate is

$$
*=\sum_{n, j} K_{4}^{n} C_{1}^{j}(j+1)^{2 n}(j !)^{-1 / 2}(4 n) !^{2} \alpha^{-\varepsilon c n^{1+o}} .
$$

Using the results: $(j+1)^{2 n} \leqq 4^{n} j^{2 n} ;(j)^{-1 / 2}<(e / j)^{j / 2} ;(4 n) !^{2}<4^{8 n} n^{8 n}$, we have that

$$
\begin{aligned}
* & <\sum_{n, j} K_{4}^{n} C_{1}^{j} 4^{n} j^{2 n}(e / j)^{j / 2} 4^{8 n} n^{8 n} \alpha^{-\varepsilon c n^{1+\delta}} \\
& =\sum_{n, j} K_{5}^{n} C_{2}^{j} j^{2 n-j / 2} n^{8 n} \alpha^{-\varepsilon c n^{1+\delta}} \\
& =\sum_{n} K_{5}^{n} n^{8 n} \alpha^{-\varepsilon c n^{1+\delta}} \sum_{j} j^{j l n C_{2} / l n j} .
\end{aligned}
$$

The sum over $j$ can be shown to be bounded by $C_{3}^{n} n^{3 n}$, and

F) $\sum_{n} C_{4}^{n} n^{11 n} \alpha^{-\varepsilon c n^{1+\delta}}$ converges.

G) The dominated convergence theorem now gives convergence of $\int_{E} h_{\sigma}$ as $\sigma \rightarrow \infty$

The constants of Lemma IV.3 will be the normalizations needed to obtain measures of total mass 1 .

Lemma IV.3.[3, pp. 13-14]. $\lim _{\sigma \rightarrow \infty}\left(\hat{T}_{k \sigma} \Omega_{0}, \hat{T}_{k \sigma} \Omega_{0}\right) e^{-X(\sigma)}=\lim _{\sigma \rightarrow \infty} c_{k \sigma}^{-2}=c_{k}^{-2}$, where $0<c_{k}^{-2}<1$.

\section{Corollary IV.4.}

$$
\lim _{\sigma \rightarrow \infty}\left(\hat{T}_{k \sigma} e^{-i a^{*}(f) / 2} \Omega_{0}, \hat{T}_{k \sigma} e^{i a^{*}(f) / 2} \Omega_{0}\right)\left\|T_{k \sigma} \Omega_{0}\right\|^{-2}=\lim _{\sigma \rightarrow \infty} L_{k \sigma}(f)=L(f)<\infty,
$$

with a uniform bound for $\{f:\|f\|<\delta\}$ and supp $f \subset\{\|k\| \leqq \varrho\}$. 
A similar sum analysis shows

Lemma IV.5. Suppose $\phi^{1}, \phi^{2} \in \mathscr{D}, \phi^{i}=\sum_{n} \phi_{n}^{i}$, supp $\left(\phi_{n}^{i}\right) \subset\{\|k\| \leqq \varrho\}$ for $i=1,2$; $\varrho>0$. Then

$$
\left(\hat{T}_{k \sigma} \phi^{1}, \hat{T}_{k \sigma} \phi^{2}\right) e^{-X(\sigma)} \leqq C\left\|\phi^{1}\right\|\left\|\phi^{2}\right\| \quad \text { for all } \sigma,
$$

where $C$ depends on $\varrho$ and on $\max \left\{i, j: \phi_{i}^{1} \neq 0\right.$ or $\left.\phi_{j}^{2} \neq 0\right\}$, but is independent of $\sigma$. It follows that

$$
\left(\hat{T}_{k} \phi^{1}, \hat{T}_{k} \phi^{2}\right)_{r} \leqq C\left\|\phi^{1}\right\|\left\|\phi^{2}\right\|
$$

Because of our normalization $L_{k \sigma}(0)=1$. We will want $L_{k}(f)={ }_{\text {df. }} \lim _{\sigma \rightarrow \infty} L_{k \sigma}(f)$ to be continuous at the origin so we need:

Lemma IV.6. [equicontinuity of $L_{k \sigma}(f)$ ]. Fix $k \in Z^{+}$. Suppose $\varepsilon>0, \varrho>0$. Then there exists a $\delta$ such that if $f\left(k_{1}\right)=0$ for $\left\|k_{1}\right\|>\varrho$ and $\|f\|<\delta$, then

$$
\left|L_{k \sigma}(f)-1\right|<\varepsilon \text { for all } \sigma \text {. }
$$

In particular, if $\left\{f_{i}\right\} \subset K$ and $f_{i} \rightarrow 0$ in $K$, then there exists an $N$ such that

$$
i>N \Rightarrow\left|L_{k \sigma}\left(f_{i}\right)-1\right|<\varepsilon \text { for all } i, \sigma \text {. }
$$

\section{Renormalized Bargmann-Segal Spaces}

Theorem V.1. Vor $f \in K$,

$$
L_{k}(f)=\lim _{\sigma \rightarrow \infty}\left(\hat{T}_{k \sigma} e^{-i a^{*}(f) / 2} \Omega_{0}, \hat{T}_{k \sigma} e^{i a^{*}(f) / 2} \Omega_{0}\right)\left\|\hat{T}_{k \sigma} \Omega_{0}\right\|^{-2}
$$

defines the characteristic function of a real-valued cylinder set measure $\eta_{k}$ on $K^{\prime}$, which is countably additive. Also,

$$
\int_{K^{\prime}} \bar{p}_{1} p_{2} d \eta_{k}=c_{k}^{2}\left(\hat{T}_{k} \hat{p}_{1} \Omega_{0}, \hat{T}_{k} \hat{p}_{2} \Omega_{0}\right)_{r}
$$

Proof. The criteria of Theorem III.1 are easily verified using the results in IV, so we have a measure $\eta_{k}$. To show the continuity condition, and therefore countable additivity, we need to examine the moments of $\eta_{k}$.

The following is known about measures on $\mathbb{R}$ : Suppose $\left\{\mu_{j}\right\}$ is a sequence of measures with moments $m_{j}^{(k)}=\int x^{k} d \mu_{j}(x)$ and suppose the sequences $m_{j}^{(k)} \rightarrow m^{(k)}$, finite. Then the limits are the moments of a measure $\mu$ such that some subsequence $\mu_{j}$, converges weakly to $\mu$ and the $m^{(k)}$ are the moments of $\mu[10, \mathrm{p} .185]$. In the case of our real measures on complex spaces, we know that the complex moments are finite; i.e. $\int \bar{p}_{1} p_{2} d \eta_{k \sigma}<\infty$ and as $\sigma \rightarrow \infty$, these expressions converge to $c_{k}^{2}\left(\hat{T}_{k} \hat{p}_{1} \Omega_{0}, \hat{T}_{k} \hat{p}_{2} \Omega_{0}\right)_{r}<\infty$. We can verify that the real moments are also finite.

Now

$$
\int_{K^{\prime}} \bar{p}_{1} p_{2} d \eta_{k \sigma}=\int_{h \in F} \bar{p}_{1}(i(h)) p_{2}(i(h)) d \eta_{k \sigma, F}(h)
$$

where $\langle f, i(g)\rangle=(g, f)$. But

$$
\int \bar{p}_{1} p_{2} d \eta_{k \sigma}=c_{k \sigma}^{2}\left(\hat{T}_{k \sigma} \hat{p}_{1} \Omega_{0}, \hat{T}_{k \sigma} \hat{p}_{2} \Omega_{0}\right) e^{-X(\sigma)}
$$


and converges as $\sigma \rightarrow \infty$ to $c_{k}^{2}\left(\hat{T}_{k} \hat{p}_{1} \Omega_{0}, \hat{T}_{k} \hat{p}_{2} \Omega_{0}\right)_{r}$, where we have used Lemma IV.3. So

$$
\lim _{\sigma \rightarrow \infty} \int \bar{p}_{1} p_{2} d \eta_{k \sigma}=c_{k}^{2}\left(\hat{T}_{k} \hat{p}_{1} \Omega_{0}, \hat{T}_{k} \hat{p}_{2} \Omega_{0}\right)_{r}
$$

So if we let $\mu_{j}=\eta_{k \sigma, F}$, then $\mu$ must be $\eta_{k, F}$ and

$$
\int \bar{p}_{1} p_{2} d \eta_{k}=\lim _{\sigma \rightarrow \infty} \int \bar{p}_{1} p_{2} d \eta_{k \sigma}
$$

where $p_{1}$ and $p_{2}$ are based on $F$, a finite dimensional subspace of $K$. The above, Chebychev's inequality, and Lemma IV.5 are used to verify the continuity condition.

We now have an isometry $E_{k}:\left\{\hat{T}_{k} \hat{p} \Omega_{0}\right\} \rightarrow H^{2}\left(K^{\prime}, d \eta_{k}\right)$ with $E_{k}\left(\hat{T}_{k} \hat{p} \Omega_{0}\right)=p / c_{k}$ and want to extend it to $\hat{T}_{k} \psi$ for more general $\psi$. Although Proposition III.5, an extension result for $\eta_{k \sigma}$, was valid for functions of arbitrary support, the extension theorem for $\eta_{k}$ considers only functions of compact support.

Proposition V.2. Fix $k \in Z^{+}$. Suppose $\phi=\Sigma \phi_{n}$, a finite sum, where $\phi_{n} \in \mathscr{D}_{n}$. Then there is an isometry $E_{k}$ from $\left\{T_{k} \mathscr{D}\right\}^{-}$into $H^{2}\left(K^{\prime}, d \eta_{k}\right)$ such that if $\hat{p}_{i} \Omega_{0} \rightarrow \phi$ in $\mathscr{F}$ and $\left\{\operatorname{supp}\left(\hat{p}_{i} \Omega_{0}\right)\right\}$ is a bounded set, then

$$
p_{\iota} / c_{k}=E_{k}\left(\hat{T}_{k} \hat{p} \Omega_{0}\right) \rightarrow E_{k}\left(\hat{T}_{k} \phi\right)
$$

in $H^{2}\left(K^{\prime}, d \eta_{k}\right)$ and

$$
\int\left|E_{k}\left(\hat{T}_{k} \phi\right)\right|^{2} d \eta_{k}=\left(\hat{T}_{k} \phi, \hat{T}_{k} \phi\right)_{r}
$$

The space $H^{2}\left(K^{\prime}, d \eta_{k}\right)$ is defined as the completion in $L^{2}\left(K^{\prime}, d \eta_{k}\right)$ of the polynomials on $K^{\prime}$. We now want to define annihilation and creation operators on $H^{2}\left(K^{\prime}, d \eta_{k}\right)$ to get (as close as possible to) a representation of the CCR.

Let $a_{k}^{*}(f)$ be multiplication by $\langle f, \cdot\rangle$, defined on

$$
\left\{\psi \in H^{2}\left(K^{\prime}, d \eta_{k}\right):\langle f, \cdot\rangle \psi \in H^{2}\left(K^{\prime}, d \eta_{k}\right)\right\} .
$$

The domain contains all polynomials. $a_{k}^{*}(f)$ is closed and for $\phi \in \mathscr{D}$,

$$
E_{k}\left(a^{*}(h) \phi\right)=\langle h, \cdot\rangle E_{k}(\phi) \text {. }
$$

Let $a_{k}(f)$ be $\left(a_{k}^{*}(\bar{f})\right)^{*}$. This operator is densely defined and closed. Unfortunately we do not know if $a_{k}$ and $a_{k}^{*}$ have a common dense domain.

We now examine the relationship between $a_{k}^{*}(f)$ and $a_{k \sigma}^{*}(f)$, and between $a_{k}(f)$ and $a_{k \sigma}(f)$.

An operator $B$, defined on a dense subset of $H^{2}\left(K^{\prime}, d \eta_{k}\right)$ is called the moving weak limit of a sequence $B_{\sigma}$ of operators, each defined on a dense subset of the corresponding $H^{2}\left(K^{\prime}, d \eta_{k \sigma}\right)$, if the domains of all $B_{\sigma}$ and $B$ include all polynomials and if for all $p_{1}, p_{2}$

$$
\int \bar{p}_{1}\left(B_{\sigma} p_{2}\right) d \eta_{k \sigma} \rightarrow \int \bar{p}_{1}\left(B p_{2}\right) d \eta_{k}
$$

Suppose $f \in \mathrm{K}$. Then $a_{k}^{*}(f)$ is the moving weak limit of $a_{k \sigma}^{*}(f)$. This result follows from V.1. 


\section{Formal Calculations}

Formally we have the following:

$$
a_{k \sigma}(f)=a(f)+\left[\left(a(f) T_{k \sigma}\right) / T_{k \sigma}\right] I_{0},
$$

where $I_{0}$ is the identity operator (compare with the discussion after III.5).

Suppose $a_{k \sigma}, a_{k}$ are defined on the polynomials. Then

$$
\begin{aligned}
\int \bar{p}_{1} a_{k}(f) p_{2} d \eta_{k} & =\int\left(a_{k}^{*}(\bar{f}) p_{1}\right)^{-} p_{2} d \eta_{k}=\lim _{\sigma \rightarrow \infty} \int\left(a_{k \sigma}^{*}(\bar{f}) p_{1}\right)^{-} p_{2} d \eta_{k \sigma} \\
& =\lim _{\sigma \rightarrow \infty} \int \bar{p}_{1} a_{k \sigma}(f) p_{2} d \eta_{k \sigma} .
\end{aligned}
$$

Since $a(f) p$ is a well-defined polynomial, we have that, formally,

$$
\left(a(f) T_{k}\right) / T_{k}=\text { moving } \underset{\sigma \rightarrow \infty}{\text { weak }} \operatorname{limit}\left(a(f) T_{k \sigma}\right) / T_{k \sigma} .
$$

CCR (formally)

$$
\begin{aligned}
\left(p^{\prime},\left[a_{k}(f), a_{k}^{*}(g)\right] p\right)_{k} “ & =" \lim _{\sigma \rightarrow \infty}\left(p^{\prime},\left[a_{k \sigma}(f), a_{k \sigma}^{*}(g)\right] p\right)_{k \sigma} \\
& =\lim _{\sigma \rightarrow \infty}\left(p^{\prime},(\bar{f}, g) p\right)_{k \sigma}=(\bar{f}, g)\left(p^{\prime}, p\right)_{k} .
\end{aligned}
$$

\section{The Inductive Limit}

Our goal is to produce a representation of the CCR which is unitarily equivalent to the Fabrey-Glimm representation. The latter is constructed by taking an inductive limit of the spaces $\hat{T}_{i} \mathscr{D}$ for $i \geqq 0$. Analogously, we take the inductive limit of the spaces $H^{2}\left(K^{\prime}, d \eta_{i}\right)$ for $i \geqq 0$, to form the space $\mathscr{H}$.

A collection of continuous linear maps $\beta_{i j}: H^{2}\left(K^{\prime}, d \eta_{i}\right) \rightarrow H^{2}\left(K^{\prime}, d \eta_{j}\right) ; i \leqq j$; $i, j \in Z^{+}$is called an inductive system if

(1) $\beta_{i i}$ is the identity map on $H^{2}\left(K^{\prime}, d \eta_{i}\right)$,

(2) $\beta_{i k}=\beta_{j k} \circ \beta_{i j}, i \leqq j \leqq k$.

Proposition VI.1. $\left\{H^{2}\left(K^{\prime}, d \eta_{i}\right), \beta_{i j}\right\}$ is an inductive system if we define $\beta_{i j}$ as follows: Let $\beta_{i j}(p)=c_{i} E_{j}\left(\hat{T}_{j}\left(\hat{T}_{i j} \hat{p} \Omega_{0}\right)\right)$, which is well-defined by V.2, and in fact equals $\left(c_{i} / c_{j}\right) T_{i j} \cdot p$, where $T_{i j}=I\left(\hat{T}_{i j} \Omega_{0}\right)$. Extend this isometry to $H^{2}\left(K^{\prime}, d \eta_{i}\right)$.

We can check that $\beta_{i k}=\beta_{j k} \circ \beta_{i j}$ by using $\hat{T}_{i j} \Omega_{0}=\lim _{s \rightarrow \infty} \sum_{n} \hat{p}_{s}^{(n)} \Omega_{0}$, where $\hat{p}_{s}^{(n)} \Omega_{0} \in \mathscr{F}$ with appropriate supports as in V.2.

Now construct the inductive limit $\mathscr{H}$ by taking the locally convex direct sum $\bigoplus_{i \geqq 0} H^{2}\left(K^{\prime}, d \eta_{i}\right)$ modulo the subspace $M$ generated by

$$
\left\{\phi_{i}-\beta_{i j}\left(\phi_{i}\right): \phi_{i} \in H^{2}\left(K^{\prime}, d \eta_{i}\right), \quad i \leqq j\right\} .
$$

Let $\beta_{j}$ be the map taking $H^{2}\left(K^{\prime}, d \eta_{j}\right)$ into $\mathscr{H}$ and let $\mathscr{F}_{r}=\left(\bigcup_{i \geq 0} \hat{T}_{i} \mathscr{D}\right)^{-}$.

For $p \in H^{2}\left(K^{\prime}, d \eta_{i}\right)$, let $\gamma_{i} p=c_{i} \hat{T}_{i} \hat{p} \Omega_{0} .\left\|c_{i} \hat{T}_{i} \hat{p} \Omega_{0}\right\|_{r}^{2}=c_{i}^{2}\left\|\hat{T}_{i} \hat{p} \Omega_{0}\right\|_{r}^{2}=\int|p|^{2} d \eta_{i}$, by V.1. Therefore $\gamma_{i}$ is an isometry and can be extended to all of $H^{2}\left(K^{\prime}, d \eta_{i}\right)$. $\gamma_{i}=\gamma_{j} \circ \beta_{i j}$. 
Theorem VI.2. There exists a unique, continuous, 1-1, onto, linear map $\gamma: \mathscr{H} \rightarrow \mathscr{F}_{r}$ that makes the diagram below commutative:

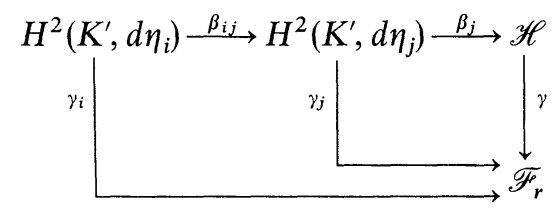

Proof. The result follows because $\mathscr{F}_{r}$ is locally convex and the $\gamma_{i}$ are $1-1$ continuous linear maps [8].

\section{Operators on $\mathscr{H}$}

Definition. For $h \in K$ define $\tilde{a}^{*}(h)$ on a dense subset of $\mathscr{H}$ as follows:

$$
\tilde{a}^{*}(h)\left(\beta_{i} p\right)=\beta_{i}\left(a_{i}^{*}(h) p\right) \quad p \in H^{2}\left(K^{\prime}, d \eta_{i}\right) .
$$

Finite sums of expressions of the form $\beta_{i} p$ are dense in $\oplus H^{2}\left(K^{\prime}, d \eta_{i}\right)$, hence dense in $\mathscr{H}$.

Proposition VI.3. $\tilde{a}^{*}(h)$ is well defined, i.e. for $i \leqq j$ we have

$$
a_{j}^{*}(h)\left(\beta_{i j} p\right)=\beta_{i j}\left(a_{i}^{*}(h) p\right) .
$$

Fabrey gives the following definition: "We say that an operator $B$, which maps a subspace of $\mathscr{F}_{r}$ into $\mathscr{F}_{r}$ is the weak limit of an operator $A$ in $F$, written $B=\lim _{\sigma} A$ if the domain of $B$ is $\bigcup_{k \geqq 0} \hat{T}_{k} \mathscr{D}$ and

$$
\left(\hat{T}_{k} \psi_{1}, B \hat{T}_{l} \psi_{2}\right)_{r}=\lim _{\sigma \rightarrow \infty}\left(\hat{T}_{k \sigma} \psi_{1}, A \hat{T}_{l \sigma} \psi_{2}\right) e^{-X(\sigma)}, \quad[3, \text { p. } 22]
$$

He proves $\left[3\right.$, p. 25] that the field operator $\phi$ has a weak $\operatorname{limit} \underset{\sigma}{\lim } \phi \subset \phi_{r}$. So we have

$$
\begin{aligned}
& a_{r}^{*}(h)=2^{-1 / 2}\left[\phi_{r}(h)-i \phi_{r}(i h)\right] \supset \lim _{\sigma \rightarrow \infty} a^{*}(h), \\
& a_{r}(\bar{h})=2^{-1 / 2}\left[\phi_{r}(h)+i \phi_{r}(i h)\right] \supset \lim _{\sigma \rightarrow \infty} a(\bar{h}) .
\end{aligned}
$$

Also, $\bigcup_{k \geqq 0} T_{k} \mathscr{D}$ is a dense set of entire vectors for $\phi_{r}(h)$, hence for the closed operators $a_{r}(h)$ and $a_{r}^{*}(h)$. The Weyl relations for $a_{r}, a_{r}^{*}$ imply that $\left[a_{r}(f), a_{r}^{*}(g)\right]_{r}=(\bar{f}, g)_{r}$.

We can verify that $\gamma\left[\tilde{a}^{*}(h)\left(\beta_{j} p\right)\right]=a_{r}^{*}(h)\left(\gamma_{j} p\right)$. Since $a_{r}^{*}(h)$ is closed, we can form the closure of $\tilde{a}^{*}(h)$.

$\tilde{a}(\bar{h})$ is defined on $\mathscr{H}$ as $\tilde{a}^{*}(h)^{*}$, or equivalently, as the operator induced on $\mathscr{H}$ by the operator $a_{r}(\bar{h})$ on $\mathscr{F}_{r}$.

We have verified everything that is needed for the following:

Theorem VI.4. The operators $\tilde{a}(h)$ and $\tilde{a}^{*}(h)$ on $\mathscr{H}$, for $h \in K\left(\mathbb{R}^{2}\right)$, define a representation of the CCR which is unitarily equivalent to the Fabrey-Glimm representation on $\mathscr{F}_{r}$ when the operators $a_{r}(h), a_{r}^{*}(h)$ on $\mathscr{F}_{r}$ are considered only for $h \in K\left(\mathbb{R}^{2}\right)$. 


\section{Formal Calculations}

We would like to compare $\tilde{a}(h)$ with the formal annihilation operators

$$
a_{i}(h)=a(h)+\left[\left(a(h) T_{i}\right) / T_{i}\right] \cdot I_{0} \quad\left(I_{0} \text { the identity operator }\right)
$$

on $H^{2}\left(K^{\prime}, d \eta_{i}\right)$, so we do the following:

Suppose the polynomials belong to the domains of all $a_{i}(h)$. Is it true, then, that $a_{j}(h) \circ \beta_{i j}=\beta_{i j} \circ a_{i}(h)$ ?

$$
a_{j}(h) \circ \beta_{i j} p=a_{j}(h) c_{i} E_{j}\left(\hat{T}_{j} \hat{T}_{i j} \hat{p} \Omega_{0}\right)=a_{j}(h) c_{i} c_{j}^{-1} T_{i j} p .
$$

As at the end of Section III, $a_{j}(h)$ would be defined on $H^{2}\left(K^{\prime}, d \eta_{j}\right)$ as the usual derivative, if $a(h) T_{j}=0$. Then we would have

And

$$
=c_{i j}\left[\left(a(h) T_{i j}\right) \cdot p+T_{i j} \cdot a(h) p\right] .
$$

$$
\beta_{i j} \circ a_{i}(h) p=\beta_{i j}\left(a(h) p+\frac{a(h) T_{i}}{T_{i}} p\right) \sim c_{i} E_{j}\left(\hat{T}_{j} \hat{T}_{i j}\left[a(h) \hat{p} \Omega_{0}+\frac{a(h) \hat{T}_{i}}{\hat{T}_{i}} \hat{p} \Omega_{0}\right]\right) .
$$

Now, if we write $T_{i}=T_{i j} T_{j}$ so

then

$$
\begin{aligned}
a(h) T_{i} & =\left(a(h) T_{i j}\right) \cdot T_{j}+T_{i j} \cdot\left(a(h) T_{j}\right) \\
& =\left(a(h) T_{i j}\right) \cdot T_{j}, \\
& =c_{i}\left[T_{i j} \cdot a(h) p+\left(a(h) T_{i j}\right) \cdot p\right] .
\end{aligned}
$$

$a(h) T_{j}=0$ is roughly equivalent to the support of $h$ being contained in

$$
\left\{k \in \mathbb{R}^{2}:\|k\| \leqq \alpha(j)\right\} .
$$

So suppose $h$ has compact support. Choose any $i$ so large that $a(h) T_{i}=0$. Then $\tilde{a}(h)\left(\beta_{i} p\right)$ can be defined as $\beta_{i}\left(a_{i}(h) p\right)$.

Acknowledgement. The author extends her thanks to Donald G. Babbitt for his help and encouragement.

\section{References}

1. Bargmann, V.: On a Hilbert space of analytic functions and an associated integral transform. Comm. Pure Appl. Math. 14, 187-214 (1961)

2. Bourbaki, N.: Éléments de Mathématique, VI. Intégration, Ch. IX, Intégration sur les Espaces Topologiques Séparés. Paris: Hermann, 1969

3. Fabrey, J.D.: Exponential representations of the canonical commutation relations. Comm. math. Phys. 19, 1-30 (1970)

4. Fabrey, J. D.: Weyl Systems for the $\left(\phi^{4}\right)_{3}$ Model, preprint.

5. Gelfand, I. M., Vilenkin, N. Ya.: Generalized functions, Vol. 4. New York: Academic Press 1964

6. Glimm, J.: Boson Fields with the $: \phi^{4}$ : interaction in three dimensions. Commun. math. Phys. 10, $1-47$ (1968)

7. Hepp,K.: Théorie de la Renormalisation. Berlin-Heidelberg-New York: Springer 1969

8. Horvath, J.: Topological vector spaces and distributions. Reading: Addison-Wesley 1966

9. Köthe, G.: Topological vector spaces I. Berlin-Heidelberg-New York: Springer 1969

10. Loève, M.: Probability theory. New York: Van Nostrand Reinhold 1963

11. Loomis, L.H.: An introduction to abstract harmonic analysis. Princeton: Van Nostrand 1953 
12. Minlos, R.A.: Generalized random processes and their extension to a measure. In: Selected translations in mathematical statistics and probability No. 3. Providence: Am. Math. Soc. 1963

13. Prohorov, Yu.V.: The method of characteristic functionals. In: Proceedings of the Fourth Berkeley Symposium on Math. Statistics and Probability, Vol. II. Berkeley and Los Angeles: Univ. of Calif. Press 1961

14. Schaefer, H.: Topological vector spaces. Berlin-Heidelberg-New York: Springer 1971

15. Segal,I.E.: Mathematical problems of relativistic physics. Am. Math. Soc., Providence, 1963

16. Segal,I.E.: Mathematical characterization of the physical vacuum for a linear Bose-Einstein field. Illinois J. Math. 6 (1962), 500-523

Communicated by A. S. Wightman

J. Kunofsky

Department of Mathematics

University of California

Berkeley, Calif. 94720, USA 